\title{
An innovative use of the K-SIR arch
}

\section{Ulrika Diana Pereira Kalia ${ }^{1}$, Prerna Raje Batham ${ }^{2, *}$, Bhakti Ramchandani ${ }^{3}$}

\author{
${ }^{1}$ Professor, Private Practive, ${ }^{2}$ Reader, Dept. of Orthodontics, Index Institute of Dental Science, Indore, Madhya \\ Pradesh, ${ }^{3}$ Senior Lecturer, Private Practive
}

\section{*Corresponding Author: Prerna Raje Batham}

Email: drprernaraje@gmail.com

\begin{abstract}
Segmental arch mechanics are always preferred mechanics of choice since a known force system is delivered to the teeth without loss of force to friction. The springs available for single canine retraction are complicated in design and time consuming to fabricate. This article presents an innovative way to use the K-SIR arch wire for single canine retraction.
\end{abstract}

Keyword: Segmental arch mechanics, Individual canine retraction, KSIR arch.

\section{Introduction}

Segmented retraction using frictionless springs is a useful method to reduce the strain on posterior anchorage. ${ }^{1}$ We are able to utilise one couple and two couple systems and an approximation of both forces and moments can be made. This allows for better use of favourable force levels and better control. The main advantage of frictionless mechanics is the establishment of well defined units of anchorage and movement segments.

In contrast, the undesirable side effects seen during canine retraction while applying friction mechanics are tipping, binding, lack of vertical control, anchorage loss and incisor extrusion.

To address these problems frictionless mechanics can be used. But the main disadvantage of segmented arch approach lies in greater complexity of the orthodontic appliance and increased chair side time of the clinician to install, adjust and maintain it. The springs available for canine retraction in segmented mechanics are $\mathrm{T}$ loop, ${ }^{2} \mathrm{PG}$ canine retraction spring ${ }^{3}$ and Ricketts canine retraction spring. ${ }^{4}$

The K-SIR or Kalra Simultaneous Intrusion and Retraction archwire when introduced, was considered to be a modification of the segmented loop mechanics by Burstone and Nanda. K-Sir arch consists of .019"x.025" TMA archwire with closed $7 \mathrm{~mm} \times 2 \mathrm{~mm}$ U-loops at the extraction sites. ${ }^{5}$

\section{Spring Design}

It was decided to modify this design by using it for individual canine retraction rather than enmasse retraction. This was done by making individual springs for each canine. The bends required to construct the KSIR Arch were kept the same. A 90 degree V bend was given at level of each $\mathrm{U}$ loop to obtain bodily movement and prevent tipping of the canine into the available space. This creates two equal and opposite moments when centrally positioned between the molar and canine and acts as a counter to the moments developed by activation of the closing loops. An increased clockwise moment is required to increase the molar anchorage and bring about canine intrusion. This is bought about by introducing a 60 degree $\mathrm{V}$ bend posterior to the centre of the inter bracket distance. A 20 degree anti rotation bend is then given just distal to the U loop to prevent mesio lingual rolling of the buccal segments caused by the force of loop activation.

\section{Activation}

As in K-SIR arch, stresses are built up while bending the wire for the springs. This is released by doing a trial activation of the springs. This also reduces the severity of the $\mathrm{V}$ bends as well. The legs of each of the springs are extended horizontally to determine the neutral position and the $\mathrm{U}$ loop will be $3.5 \mathrm{~mm}$ wide in this position. On activation of $3 \mathrm{~mm}$ the distance between the medial and distal legs is considerably reduced. On initial activation the retraction force creates larger tipping moments in comparison to the opposing moments generated by the $\mathrm{V}$ bends. This brings about a controlled tipping of the canine into the extraction space and once deactivation of the loops occur the moment to force ratio increases causing bodily movement of the canine followed by root movement. This allows for activation to be done after every six to eight weeks till the space is closed.

\section{Case Report}

A 23 year old male patient was referred to the Department of orthodontics and dentofacial orthopaedics from the Department of Prosthodontics for the consolidation of spaces distal to maxillary canines. On clinical examination it was noted that the maxillary and mandibular incisors and maxillary right first molar were missing. The maxillary canines were forwardly placed and extruded. For prosthetic replacement the patient required orthodontic treatment to correct the position of the maxillary canines and to correct the malalignment in the lower dentition. A 3M Unitek Gemini metal bracket kit with 0.022 slot was used and banding of molars was done using upper double and lower single 0.022" X 
0.028 " tubes. The maxillary canines and the lower dentition were completely bonded and 0.014" Niti was placed for alignment of the lower arch. The Modified KSIR spring was placed on both the maxillary canines (Fig. 1). Initially the springs were placed but not activated. Activation was done after 1 month and then subsequent activations were done every six to eight weeks until the space distal to the canines were closed and the canines were intruded (Fig. 2). Once the maxillary canines were distalized the first and second maxillary premolars were bonded and continuous wires were placed for arch alignment and levelling. The wire sequencing for the maxillary and the mandibular arches were as follows- 0.014 " Niti, 0.016" Niti, 0.017 X 0.025" HANT and 0.019 X 0.025 " S.S. Debonding (Fig. 3) was done and a functional retainer was placed with missing teeth replaced till the department of Prosthetics was able to deliver their prosthesis to the patient. Pre treatment and post treatment OPGs (Fig. 4 and Fig. 5) which were taken as a part of routine patients records showed the position of the canine before and after the use of the springs.

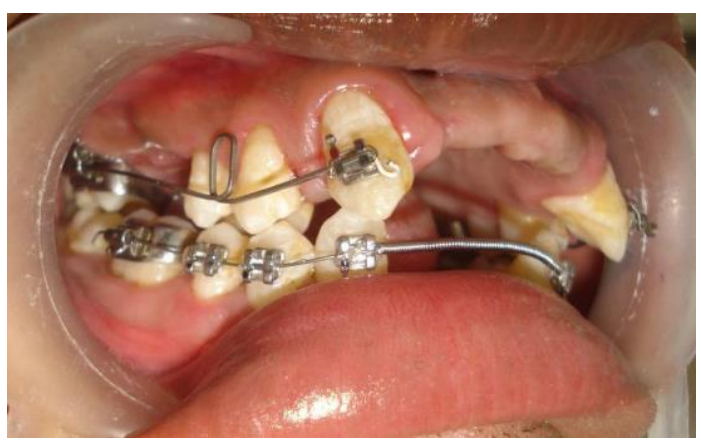

Fig. 1: Individual intrusion and retraction spring at the start of canine retraction

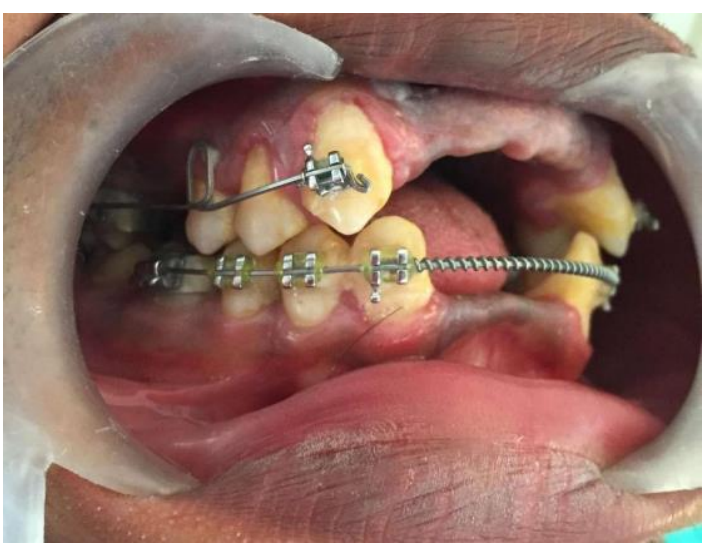

Fig. 2: Individual intrusion and retraction spring at the end of canine retraction

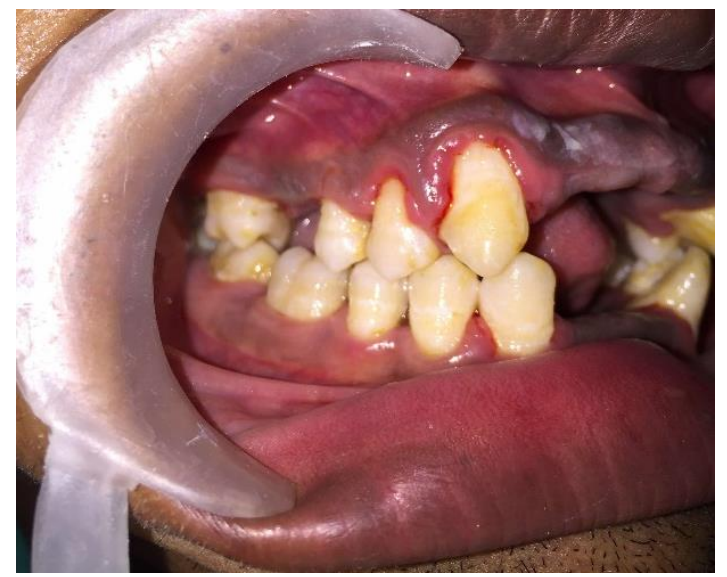

Fig 3: Post debonding

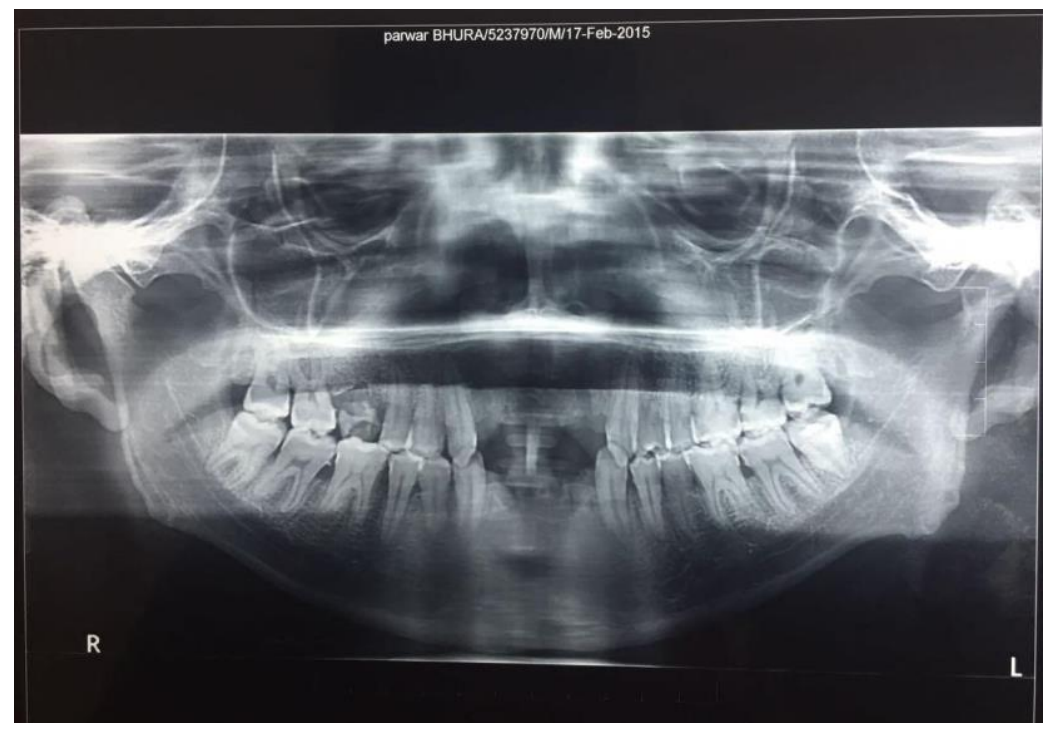

Fig. 4: Pre treatment OPG 


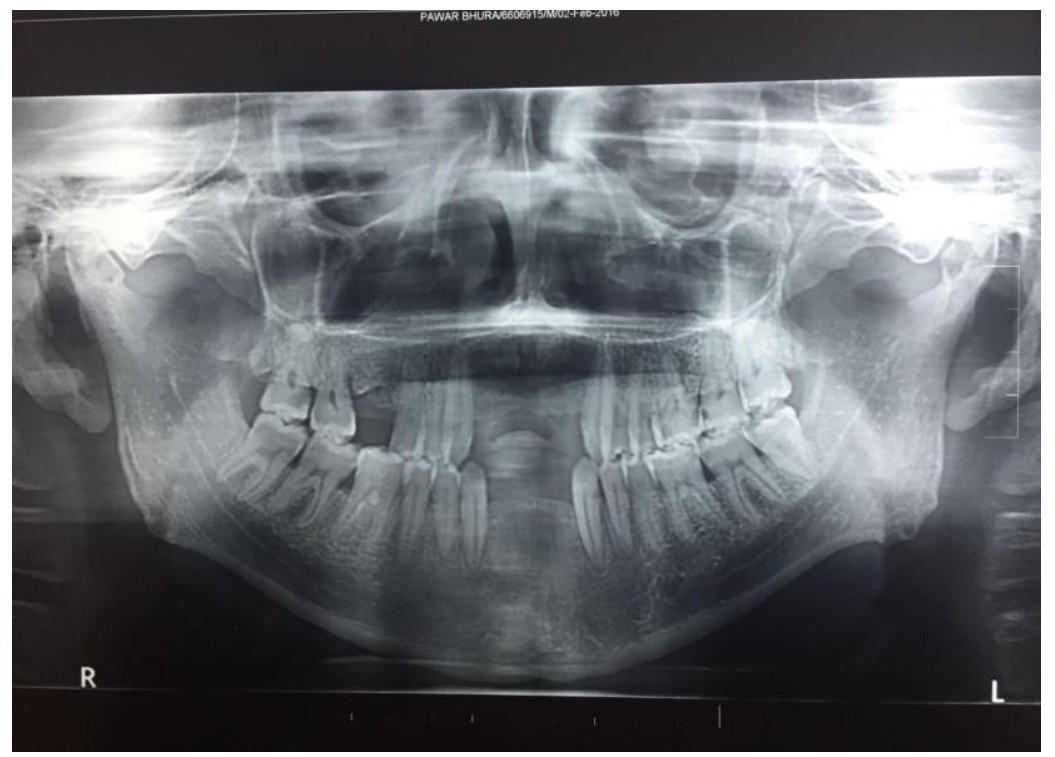

\section{Fig. 5: Post treatment OPG}

\section{Discussion}

The main indication of the K-SIR arch wire was to retract the anterior teeth in a first premolar extraction patient who had deep overbite and excessive overjet and would require both intrusion of the anterior teeth and maximum molar anchorage. The arch wire would require a few modifications to be used in moderate and minimum anchorage cases with varying degrees of overbite. Another positive feature of the K-SIR arch was its ease in fabrication, patient comfort and lack of tissue impingement. The use of TMA for the construction of this arch wire provided with sufficient strength to resist distortion but enough stiffness to generate the required moments. Together the K-SIR arch could provide low forces, low load deflection rate and a range of activation that allowed the appliance to continue closing the space.

In the case described above the maxillary canines required intrusion and retraction to correct their position within the arch so as to aid in prosthetic replacement of the missing anterior teeth. By modifying the K-SIR arch into a spring we were able to benefit from the advantages of segmental mechanics and that of the K-SIR arch and were able to simultaneously retract and intrude the canines in question for successful prosthetic replacement.

\section{Conclusion}

By converting the K-SIR arch into a spring we were able to achieve a successful outcome by understanding the biomechanics involved and using it to our advantage.
3. Gjessing P. Biomechanical design and clinical evaluation of a new canine retraction spring. Am J Orthod Dentofac Ortho. 1987;5:353-362.

4. Ricketts R. Development of retraction sections. Foundations of Orthodontic Research Newsletter. 1974;5:41-44.

5. Kalra V. Simultaneous intrusion and retraction of the anterior teeth. J Clin Orthod. 1998;32:535-540.

\section{References}

1. Proffit W.R, Fields H.W, Sarver D.M. Contemporary

Orthodontics. 4th ed. St. Louis: Mosby Co; 2007.

2. Burstone CJ, Koenig HA. Optimising anterior and canine retraction. Am J Orthod Dentofac Orthop. 1976;70:1-19. 